The age of these patients ranged from $20-44$ years. The patients presented with cord-like thickening on penis within 24-48 hours after a prolonged sexual act with or without an intercourse. Seventeen patients had history of one or more episodes of STDs at presentation or in the past. Histopathological specimens showed prominent vessels with plump endothelial cells and thickened blood vessel walls. Occasional vessel showed complete occlusion of its lumen. Doppler US done in 10 patients showed dorsal vein thrombosis without the flow signals in this area. Patients were treated with counselling, abstinence and paracetamol. Thirty patients had resolution of the swelling by 6-8 weeks with treatment only two patient required surgery. No recurrence or erectile dysfunction was noted in any of the patients in the follow up from 2 to 8 years.

Conclusions Penile Mondor's disease has a favourable evolution and functional prognosis; although various etiologies have been proposed; trauma caused by sexual intercourse or masturbation is the main etiologic factor. Doppler US is a non invasive diagnostic modality helpful in both diagnosis and follow-up, however further analysis of Doppler US findings in a larger number of cases needs to be done to elucidate the hemodynamic changes in this not so rare entity.

\section{P2.131 A CLINICO-AETIOLOGICAL AND ULTRASONOGRAPHIC STUDY OF PEYRONIE'S DISEASE}

doi:10.1136/sextrans-2013-051184.0395

B Kumar. Silver Oaks Multi Specialty Hospital, Mohali- Punjab, India

Bhushan Kumar, Murlidhar, Tarun Narang

Background Peyronie's disease (PD) is caused by progressive fibrotic scarring of the tunica albuginea resulting in curvature or other deformities of the erect penis. PD is a psychologically and physically devastating disorder that manifests in middle-aged men. Although long recognised as an important clinical entity of the male genitalia, the aetiology of this disease is poorly understood.

Methods in this retrospective analysis we studied the epidemiology and clinical presentation of PD cases presenting to the dermatology out patient during Jan2001 - Dec2010. Diagnosis of PD was based on medical and sexual history, physical examination and imaging examinations: ultrasound, and colour Doppler ultrasound. Results Fifty-six men with PD presented during the period of ten years. Their ages ranged from 23 to 70 years. Most of them presented during the early phase of the disease. Most common presenting complaint was penile curvature in $80.95 \%$ followed by pain on erection in $66.66 \%$. History of penile trauma was revealed by $9.52 \%$ patients. Hypercholesterolemia (60\%), hypertension (33.3\%) and asymptomatic hyperuricemia $(28.34 \%)$ were the most common risk factors.32 patients with $\mathrm{PD}$ were studied by ultrasonography. Ultrasonogram was more accurate than clinical assessment in delineating the extent of lesions. In one-third of the patients, sonography demonstrated the plaques to be more extensive than had been detected by clinical examination.

Conclusions PD is a fibrotic wound-healing disorder involving the tunica albuginea of the corpora cavernosa leading to a variety of deformities of the genitalia which are associated with significant sexual dysfunction. The clinical symptoms and signs in our study were, in general, similar to those found in the previous studies. Higher incidence of hypertension and hypercholesterolemia in patients with PD may also be to an extent due to patients being in an older age group. However, it is difficult to explain asymptomatic hyperuricemia.

\section{P2.132 PHARYNGEAL AND CONJUNCTIVAL CHLAMYDIA TRACHOMATIS INFECTIONS: CHICKEN OR EGG?}

doi:10.1136/sextrans-2013-051184.0396

G Haidari, J A White. Guys \& St Thomas' NHS Foundation Trust, London, UK
Background Chlamydial inclusion conjunctivitis caused by genital serovars of Chlamydia trachomatis (CT) is well-recognised, and usually thought to result from auto-inoculation from genital CT infection or direct sexual contact. In this case series we review nine patients with co-existing pharyngeal and conjunctival CT infection and hypothesise on the relationship between the two conditions.

Methods Retrospective analysis of 9 patients with pharyngeal and conjunctival CT infection. Diagnoses were made based on clinical findings and the detection of CT RNA by the Aptima Combo2 assay (Gen-Probe).

Results We found 9 cases of coexisting pharyngeal and conjunctival infection in men: 8 were men who have sex with men (MSM) and 1 was heterosexual. All but one MSM had participated in both receptive and insertive oral sex, with the final patient reporting insertive oral sex and rimming.

All patients were symptomatic with unilateral conjunctivitis; one had symptoms bilaterally. Four of the 9 patients had a normal anogenital examination, and only 1 patient had a sore throat. Six of nine patients also had rectal CT infection, with 1 equivocal rectal CT result. Only two patients had co-existing urethral CT infection. Two patients had solely pharyngeal CT, including the heterosexual man.

Conclusions Whilst traditionally thought to be a result of autoinoculation from genital CT infection, we speculate that pharyngeal CT infection might be a more common source, or even a sequela, of CT conjunctivitis - at least in MSM. Alternatively, CT detected in the throat might be secondary to drainage of lacrimal fluid from a CT-infected eye. As CT conjunctivitis might be treated in isolation without comprehensive CT screening, or at most, with genital CT screening alone, we feel pharyngeal CT testing is indicated in all patients with CT conjunctivitis. The role of the nasolacrimal duct as a potential two-way conduit of infection requires further investigation.

\section{P2.133 PLASMA CELL BALANITIS (PCB). A CLINICO-PATHOLOGIC STUDY OF 132 CASES}

doi:10.1136/sextrans-2013-051184.0397

B Kumar. Silver Oaks Multi Specialty Hospital, Mohali-Punjab, India

Bhushan Kumar, Tarun Narang, B.D. Radotra

Background PCB also knows as Zoon balanitis, is a benign asymptomatic but chronic and erosive inflammatory condition of glans penis and prepuce that generally affects uncircumcised men in later years. Clinical presentation involves usually a single, shiny, well defined reddish patch. Disease may persist for years which adversely affects the quality of life of the individuals.

Methods 132 patients with a clinical diagnosis of PCB were studied between 2001-2010. Biopsy was done in patients who agreed for the procedure. Patients who did not agree for circumcision were prescribed fluticasone cream or tacrolimus $0.1 \%$ and were followed up. Results The age of the patients ranged from 24-70 years. Majority of patients had symptoms for more than 6 months. Lesions involved prepuce and glans in majority of patients; $82(62.12 \%)$, prepuce only in $24(18.18 \%)$ and glans only in $26(19.69 \%)$. Tissue for histopathology was available in 115 patients. Histologically, epidermal edoema, a dense upper dermal band of chronic inflammatory cells, including many plasma cells, dilated capillaries, extravasated red blood cells and hemosiderin deposition, were seen. In all the 115 cases PCB was successfully treated by circumcision. Remaining patients were treated with fluticasone or tacrolimus $0.1 \%$. The period of follow up ranged from $2-7$ years. Circumcised patients did not develop any recurrences. However the patients treated with fluticasone and tacrolimus had a course of relapses and remissions. Only one patient developed erythroplasia of Query at during the follow up. 
Conclusions PCB is a benign condition, with characteristic clinical and histopathological features. Although various treatment modalities have been used, circumcision remains the treatment of choice. PCB is an expression of dysfunctional foreskin, moreover the curative effect of circumcision in $100 \%$ of our patients suggests that it is a nonspecific reactive balanitis caused by a disturbed "preputial-ecology.

\section{P2.134 OPHTHALMIC AND OTOLARYNGOLOGICAL MANIFESTATIONS OF SYPHILIS IN PATIENTS WITH HIV}

doi:10.1136/sextrans-2013-051184.0398

II A Orlova, II O Smirnova, 'A V Korobko, "Y G Petunova, ${ }^{2 T}$ S Smirnova, ${ }^{2} \mathrm{~V} U$ Dudko, ${ }^{2}$ V Litvinenko, ${ }^{2} \mathrm{~A}$ B Piryatinskaya, ${ }^{2} \mathrm{~N}$ V Smirnova. ${ }^{1}$ Saint-Petersburg State University, Medical Faculty, Saint-Petersburg, Russian Federation; ${ }^{2}$ Saint-Petersburg Dermatovenerologic Dispensary, Saint-Petersburg, Russian Federation

Background Recently noteworthy aspect of the problem is associated with an increase in cases of syphilis as part of mixed infection including HIV. Mixed infection syphilis and HIV alters the clinical picture and prognosis of both diseases.

Methods 316 patients with early forms of syphilis were treated in city hospital in St. Petersburg in 2006-2010. The study group included 153 patients with syphilis + HIV (10 - primary syphilis, 117 - secondary syphilis, 26 - early neurosyphilis). Control group 163 patients with syphilis (14 - primary syphilis, 115 - secondary syphilis, 34 - early neurosyphilis). Ophthalmic and otolaryngological manifestations of syphilis were estimated.

Results Ophthalmic and otolaryngological manifestations of syphilis were diagnosed in $18(11.8 \%)$ patients of study group and 4 $(2.5 \%)$ - control group $(p<0.05) .12$ and 2 patients with secondary syphilis, 6 and 2 patients with early neurosyphilis of study and control group respectively.

In none of the groups ophthalmic and otolaryngological manifestations in patients with primary syphilis were not determined. Specific papillitis $(5(3.3 \%))$ and specific panuveit $(5(3.3 \%)$ as well as specific laryngeal neuropathy $(1(0.6 \%))$ and specific bilateral hypoacusis $(1(0.6 \%))$ were identified only in study group. Specific anterior uveitis was detected in both groups (1 and 2 patients), specific angina (3 and 1 patients) and specific laryngitis (monochorditis) (2 and 1 patients) of study and control group respectively.

Among 18 patients of study group with ophthalmic and otolaryngological manifestations were 12 cases of secondary syphilis and 6 - early neurosyphilis. 1 patient showed simultaneous ophthalmic, otolaryngological disorders and diffuse neurological symptoms.

Conclusion Clinical manifestations of syphilis were more varied in patients with HIV. Ophthalmic and otolaryngological manifestations of syphilis were more common in patients with mixed infection syphilis + HIV, which may indicate a more severe course of syphilis in these patients.

\section{P2.135 SITUATION OF SEXUALLY TRANSMITTED INFECTIONS IN MOST HIV/AIDS RISK POPULATIONS (MARPS) IN 8 PROVINCES IN VIETNAM}

doi:10.1136/sextrans-2013-051184.0399

${ }^{1} \mathrm{~K}$ H Tran, ${ }^{1} \mathrm{H}$ D Nguyen, ${ }^{1} \mathrm{P}$ M T Pham, ${ }^{1} \mathrm{H}$ Quach, ${ }^{2} \mathbf{T}$ T Vu, ${ }^{2} \mathrm{~N}$ T T Do, ${ }^{2} \mathrm{~A} 0$ Chu. ${ }^{1}$ National hospital of dermatology and venereology, ha noi, Viet Nam; ${ }^{2}$ Viet Nam - USA collaboration HIVIAIDS Project on Prevention and Care (LIFE-GAP project) in Vietnam, Ministry of Health, ha noi, Viet Nam

Background Many studies show, STIs have a closed relation with HIV. STI increases the risk of getting HIV, HIV infection makes STIs more difficult to diagnose, treat. From 2011-2012, STI services were provided to most-at-risk populations (MARPs) in 8 provinces in Vietnam by the support from CDC/PEPFAR. The MARPs included commercial sex workers (CSWs), men have sex with men (MSM) and injecting drug users (IDUs).
Method A descriptive cross sectional study of 5187 MARPs in 8 provinces in Vietnam was undertaken from 10/2011-9/2012 to determine the prevalence of STIs in specific MARPs.

Results Among 5187 MARPs screened for STIs, 4,119 MARPs (79.4\%) had STIs. Among STIs patients recorded, CSW had the highest proportion (83\%), followed by IDUs (16\%) and MSM only $1 \%$.

Among STI patients, 59.7\% ( $\mathrm{n}=2461)$ were diagnosed by syndromic approach and $40.3 \%$ by etiologic approach. In the group of syndromic diagnosis: Among 2,105 CSWs recorded, 51.7\% had vaginal discharge and 7.9\% had genital ulcer. Among 353 IDUs, $89.4 \%$ had urethral discharge and the proportion of genital ulcer was accounted for only $0.6 \%$. Regarding MSM, all of them have urethral discharged $(n=3)$.

In the group of etiologic diagnosis: Among 1,313 CSWs tested to detect STI, $28.9 \%(n=379)$ had bacterial vaginitis, $22.7 \%(n=298)$ had vuvovaginal Candidiasis, $2.2 \%(\mathrm{n}=30)$ had genital Chlamydia infection, and $1.2 \%(\mathrm{n}=16)$ had vaginal Trichomoniasis. $26.9 \%$ $(n=352)$ of the CSWs had genital wart. Among 303 IDUs, 32.6\% $(\mathrm{n}=100)$ had genital wart, $17.9 \%(\mathrm{n}=55)$ had gonorrhoea and $11.1 \%(n=41)$ had genital HSV infection. Among 42 MSM, 85.7\% $(\mathrm{n}=36)$ had genital warts and, $7.1 \%(\mathrm{n}=3)$ had gonorrhoea.

Conclusion Vaginal discharge syndrome was the most common syndrome among CSWs, urethral discharge was the most common syndrome among IDUs. Among CSWs, the most common STIs were bacterial vaginitis, HPV. Among IDUs and MSM, the most common STIs was HPV.

\section{P2.136 SEXUAL TRANSMISSION INFECTION SITUATION IN PEOPLE LIVING WITH HIV (PLHIV)IN 6 PROVINCES IN VIETNAM FROM 2008-2011}

doi:10.1136/sextrans-2013-051184.0400

${ }^{1} \mathrm{~K}$ H Tran, ${ }^{\mathbf{P}} \mathbf{M}$ T Pham, ${ }^{1} \mathrm{H} \mathrm{D}$ Nguyen, ${ }^{1} \mathrm{H}$ T Quach, ${ }^{2} \mathrm{~T} T \mathrm{Vu},{ }^{2} \mathrm{~N}$ T Do, ${ }^{2} \mathrm{~A} O \mathrm{Chu}$ ${ }^{1}$ National hospital of dermatology and venereology, ha noi, Viet Nam; ${ }^{2}$ Viet Nam - USA collaboration HIVIAIDS Project on Prevention and Care (LIFE-GAP project) in Vietnam, Ministry of Health, ha noi, Viet Nam

Background Multiple studies have suggested that STIs (sexual transmission infections) are important cofactors in the transmission and acquisition of HIV infection. Thus control of one may have beneficial effects on the control of the other.

Method A descriptive cross sectional study of 2059 PLHIV and STIs were undertaken at 6 at adult HIV outpatient clinics in Vietnam from $10 / 2008$ to $11 / 2011$ to determine the STIs situation among PLHIV.

Results Among 2059 PLHIV and STIs 48.2\% ( $\mathrm{n}=991)$ were male, $51.8 \%(n=1068)$ were female. The majority of PLHIV with STIs $(\mathrm{n}=2010,97.6 \%)$ are the ages of from 15 to 49 years, only $2.4 \%(n=49)$ of patients over 49 years. $65.6 \%(n=1350)$ of patients are diagnosed STI by etilogic approach. $34.4 \%(n=709)$ of STI/HIV patients were diagnosed by syndromic approach. Among 336 male patients ( $n=47.4 \%$ ) diagnosed with STIs by syndromic approach: $(80.1 \%)(\mathrm{n}=269)$ patients had urethral discharge syndrome, $(19.4 \%)(n=65)$ patients had genital ulcer syndrome. Among 373 female patients $(n=52.6 \%)$ diagnosed with STIs by syndromic approach, $\mathrm{n}=190(50.9 \%)$ patients had vaginal discharge syndrom, $\mathrm{n}=148(39.7 \%)$ had abdominal pain syndrome. Of 655 male patients etiologically diagnosed of STI, $65.7 \%(\mathrm{n}=430)$ had genital warts with human papillomavirus (HPV), $\mathrm{n}=73(11.2 \%)$ had genital ulcers due to Herpes simplex virus (HSV), $\mathrm{n}=38(5.8 \%)$ had gonorrhoea, $\mathrm{n}=22(3.4 \%)$ had Chlamydia trachomatis $3 \%$. Of 695 female patients, $\mathrm{n}=295$ (42.4\%) had warts due to HPV, $\mathrm{n}=121(17.4 \%)$ had fungal vaginitis due to Candida, $\mathrm{n}=117(16.8 \%)$ had bacterial vaginalis and $\mathrm{n}=79(11.4 \%)$ had ulcers due to HSV. 